\title{
Agronegocios
}

\section{Factores de riesgo operacional en agricultura: evaluación por medio del método de Proceso Jerárquico Analítico (AHP): Estudio de caso en el cultivo de café}

Operational risk factors in agriculture: Evaluation through the Analytical Hierarchical Process (AHP) method: Case study on the coffee crop

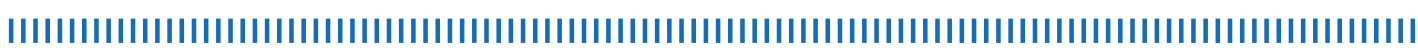

\section{Diego Fernando Quirós Badilla ${ }^{1}$ Johanna Solórzano Thompson² Javier Paniagua Molina ${ }^{3}$} Fecha de recepción: 23 de marzo, 2020 Fecha de aprobación: 05 de junio, 2020

Vol.6 $\mathrm{N}^{\circ} 2$ Julio- diciembre 2020 Quirós, D., Solórzano, J. y Paniagua, J. (2020). Factores de riesgo operacional en agricultura: evaluación por medio del método de Proceso Jerárquico Analítico (AHP): estudio del caso en el cultivo de café. Revista e-Agronegocios, 6(2). https://revistas.tec.ac.cr/index.php/eagronegocios/

article/view/5213

DOI: https://doi.org/10.18845/ea.v6i2.5213

${ }^{1}$ Universidad de Costa Rica. Costa Rica. Docente Escuela de Economía Agrícola y Agronegocios. Correo electrónico: diego.quirosbadilla@ucr.ac.cr.

2 Universidad de Costa Rica. Costa Rica. Docente Escuela de Economía Agrícola y

Agronegocios. Correo electrónico: johanna.solórzano@ucr.ac.cr. 


\section{Resumen}

El objetivo de este estudio fue determinar un valor ponderado de la importancia de las fuentes de riesgo operacional en la agricultura, así como de los factores que las componen. Además, se identificaron eventos de riesgo operacional aplicado a un estudio de caso en producción de café (Coffea arábica) en Costa Rica. Se utilizaron paneles para la aplicación del método de proceso jerárquico analítico (AHP) con la meta de obtener la ponderación de importancia con respecto al riesgo de las fuentes y factores de riesgo operativo, así mismo, se implementó un segundo panel bajo la misma estructura obtenida por el método AHP para la determinación de eventos de riesgo en el cultivo del café. Los resultados obtenidos determinaron que, en general, los factores climáticos y ambientales son los más riesgosos (55.96\%), seguido de los procesos productivos $(24,95 \%)$, mientras que la gestión del recurso humano y de las tecnologías productivas poseen el mismo porcentaje (9,55\%). Se obtuvo una ponderación para cada factor de riesgo propuesto y se logró identificar 79 eventos de riesgo en el cultivo de café bajo esta misma estructura. Este estudio permitió priorizar de manera ponderada los componentes del riesgo operativo en sistemas de producción agrícola, concepto que no se había analizado anteriormente en el sector agrícola; y permitió la generación de una estructura sólida para la identificación de eventos de riesgo específicos.

Palabras clave: Gestión de riesgo, Coffea arabica, proceso productivo, factores ambientales.

\section{Abstract}

The objective of this study was to determine a weighted value of the importance of the sources of operational risk in agriculture, as well as the factors that compose them. Besides, operational risk events applied to a case study in coffee production (Coffea arabica) in Costa Rica were identified. The panels were used for the application of the analytical hierarchy process method (AHP) to obtain the weight of importance concerning the risk of the sources and operational risk factors, likewise, a second panel was implemented under the same structure obtained by the AHP method for the determination of risk events in the selected crop. The results obtained determined that, in general, climatic and environmental factors are the riskiest (55.96\%), followed by production processes (24.95\%), while human resource management and production technologies have the same percent (9.55\%). Weight was obtained for each proposed risk factor and 79 risk events were identified in the coffee crop under this same structure. This study allowed us to prioritize the components of operational risk in agricultural production systems, a concept that had not been previously analyzed in the agricultural sector; and allowed the generation of a solid structure for the identification of specific risk events.

Key words: Risk management, Coffea arabica, production process, environmental factors. 


\section{Introducción}

La evaluación del riesgo en actividades agrícolas se ha convertido en un tema prioritario para la toma de decisiones en los sistemas productivos (Barnett \& Coble, 2008; Kahan, 2013; Komarek et al., 2020; Lagerkvist, 2005), principalmente por ser un sector altamente vulnerable a la mayoría de tipos de riesgo, como los riesgos financieros, de mercado, políticos-legales, operacionales y climáticos-ambientales (Baquet et al., 1997; Kahan, 2013; Komarek et al., 2020).

Existen diversos estudios que demuestran preferencias en las medidas de los agricultores con respecto al riesgo (Guan \& Wu, 2017, 2017; Hansson \& Lagerkvist, 2012; Young, 1979), o bien, como su percepción del riesgo puede ser un factor importante en la toma de decisiones en las actividades operativas de la producción (Bergfjord, 2009; Fahad et al., 2018; Flaten et al., 2005; Sulewski \& Kłoczko-Gajewska, 2014; Wilson et al., 1988), sin embargo, no existen análisis de manera integral en el sector agrícola con la definición de riesgo operativo u operacional, como si existe en el contexto bancario y empresarial.

No obstante, en un sistema de medición de riesgo agrícola y con escenarios sin datos o con información insuficiente, surge la necesidad de priorizar las fuentes y factores de riesgo presentes en las operaciones de los sistemas de producción agrarios. Para este fin y tomando la categorización de riesgo operativo establecida por Quirós (2018), se pueden jerarquizar dichas variables para integrarlas a un sistema de evaluación por medio de matrices de calor (Quirós-Badilla, 2019), donde con futura investigación se pueden adicionar indicadores y sub-indicadores para ser utilizados en la toma de decisiones.

El presente documento propone determinar un valor ponderado de la importancia de fuentes de riesgo operacional en la agricultura, así como de los factores que las componen, además de la identificación de eventos de riesgo operacional específicos aplicado a un estudio de caso en producción de café (coffea arábica), por medio del uso de método de Proceso Jerárquico Analítico (AHP), utilizado ampliamente en diversos estudios para priorizar un objetivo complejo frente a criterios múltiples en situaciones empíricas (Dos Santos et al., 2019; Zyoud \& Fuchs-Hanusch, 2017). La metodología también es utilizada por Toledo et al (2011) para jerarquizar tipos de riesgo presentes en la agricultura dentro de un contexto de riesgos generalizados y por Badri et al (2013) para determinar y ponderar el conjunto de riesgos presente en un proyecto de minería. Ambos objetivos se llevaron a cabo por medio de consulta con expertos en gestión de riesgos en agricultura, asi como con expertos en la producción de café respectivamente.

\section{Referente Teórico}

El riesgo se define como "la posibilidad de adversidad o pérdida"(Harwood et al., 1999, p. 2). La literatura menciona de manera recurrente 5 tipos fuentes de riesgo en la agricultura los cuáles son los riesgos de producción, financieros, mercado, institucional y personas (Komarek et al., 2020), sin embargo, dentro del contexto agricola y pecuario no se menciona de mane- 
ra recurrente el riesgo operativo como una fuente de riesgo, otros autores como Miller et al (2004) categorizan el riesgo de la producción como el riesgo del negocio y el riesgo financiero en la empresa agrícola.

De esta forma, en concordancia con el concepto bancario y empresarial, se utiliza la definición de riesgo operacional establecida por Quirós (2018, p. 19) de manera reciente para agricultura: "El riesgo de sufrir daños y pérdidas en la producción debido a inadecuaciones en el proceso productivo, la gestión del recurso humano, la gestión de las tecnologías de producción y la exposición a factores ambientales no controlables".

Existen diversos métodos para la evaluación del riesgo operativo en otros sectores de la economía (banca, empresarial, industrial), por ejemplo: matrices de calor (Palma Rodríguez, 2011; Quirós-Badilla, 2019) o el establecimiento de indicadores básicos (Arbeláez et al., 2006; Delfiner \& Pailhé, 2009; Girdžiūtè, 2012). Si bien es cierto, el presente estudio no establece una medición del riesgo operativo, pero si una ponderación de sus fuentes y factores, para ser utilizados posteriormente en análisis de evaluación.

La gestión de riesgos es el proceso por el cual se identifican las amenazas, se ponderan los riesgos encontrados, se analizan, se evalúan y se toman medidas de control para la reducción o mitigación de los posibles impactos (Rossinskaya et al., 2019). Un método utilizado para obtener una jerarquización y ponderar criterios es el Proceso Jerárquico Analítico (AHP) propuesto en los años 80 por Saaty (2008), el cual es una herramienta multicriterio que se basa en la comparación pareada de criterios o alternativas mediante una escala definida para priorizar la resolución de diferentes problemáticas o situaciones, explicado de manera más amplia en la sección de metodología; el cuál ha sido utilizado en agricultura para analizar riesgos (He et al., 2016; Li et al., 2013; Lu et al., 2016; Srinivasa Rao et al., 2019; Toledo et al., 2011)

La "identificación de nuevos eventos o re-especificación de eventos" (Miller et al., 2004, p. 20) es parte fundamental de un sistema de gestión de riesgos en la agricultura, una vez definida una ponderación o jerarquización de las fuentes y factores de riesgos presentes en el sistema productivo, siendo además, un proceso crítico para determinar cuáles riesgos puede gestionar la empresa agrícola, y qué medidas se deben tomar para aquellos riesgos sobre los cuales no se posee la capacidad de atención (Yalçinkaya, 2014).

\section{Metodología}

Se trabajó un diseño de investigación de estudio de caso, es decir, se enfoca en el examen y análisis en profundidad de las variables consideraras para este caso, las cuatro fuentes del riesgo operacional y su contexto de manera sistémica (ya que se enfoca en 4 áreas fundamentales dentro de una producción agrícola) y holística (porque busca explicar cada criterio como un todo dentro del concepto de riesgo operativo). Además, se trabaja con el método AHP, para lograr una ponderación de importancia con respecto a las variables de análisis. 
Para la resolución de los dos objetivos propuestos, se realizaron dos paneles de expertos; el primero con 11 profesionales en agronomía y gestión de riesgos en agricultura, cinco de ellos son expertos internacionales y seis nacionales, para la aplicación del método AHP y obtener así la ponderación de importancia con respecto al riesgo de las fuentes y factores de riesgo operativo. El segundo panel se realizó con cinco expertos en el cultivo de café de diferentes regiones de Costa Rica, con el uso del modelo de criterios y sub-criterios de riesgo operativo obtenido a partir del método AHP, para identificar eventos específicos en una primera etapa y que de manera generalizada pueden representar riesgos dentro de cada factor de riesgo operativo propuesto.

\section{Método de Análisis Jerárquico Analítico}

Este método consiste en dar respuesta a problemas complejos con criterios múltiples, cuyo proceso requiere de opiniones o evaluaciones subjetivas. Además de otorgar una importancia relativa a cada uno de los factores basado en el juicio de expertos, para comparar estas escalas de prioridad de manera pareada, es decir, cada criterio y subcriterio se compara uno con otro por medio de matrices. (Saaty, 2008)

Las comparaciones, por lo general, se basan en la escala numérica propuesta por Saaty (2008), que va de 1 a 9, donde el valor 1 define que ambas alternativas son igualmente importantes de acuerdo al objeto de estudio y 9 indica el grado más alto de preferencia en la alternativa seleccionada con respecto a la comparada. Cuando se prefiere la segunda alternativa, el número está precedido por el signo negativo o bien por la inversa del número, el criterio numérico se detalla en el cuadro 1:

Cuadro 1. Escala de comparación de preferencia

\begin{tabular}{cl}
\hline Valor & \multicolumn{1}{c}{ Comparación de preferencia } \\
\hline $\mathbf{9}$ & Extremadamente más riesgoso \\
\hline $\mathbf{8}$ & Entre muy fuertemente más riesgoso y extremadamente más riesgoso \\
\hline $\mathbf{7}$ & Muy fuertemente más riesgoso \\
\hline $\mathbf{6}$ & Entre fuertemente más riesgoso y muy fuertemente más riesgoso \\
\hline $\mathbf{5}$ & Fuertemente más riesgoso \\
\hline $\mathbf{4}$ & Entre moderadamente más riesgoso y fuertemente más riesgoso \\
\hline $\mathbf{3}$ & Moderadamente más riesgoso \\
\hline $\mathbf{2}$ & Entre igualmente riesgoso y moderadamente más riesgoso \\
\hline $\mathbf{1}$ & lgualmente riesgoso \\
\hline
\end{tabular}

Fuente: Adaptado de Saaty (2008). 
Las respuestas se resumen en una matriz (nxn) A = (aij), donde cada elemento aij = wi $/ \mathrm{wj}$, representa las ponderaciones de la alternativa i con respecto a j, y w es el vector prioritario construido a partir de las respuestas de los entrevistados. Esta matriz se expresa en la ecuación 1, donde dicha matriz se repite para cada evaluación de criterios que se estén analizando, luego de nuevo para cada subcriterio:

$$
A=(a i j)=\left[\begin{array}{ccc}
1 & \cdots & w 1 / w n \\
\vdots & \ddots & \vdots \\
w n / w 1 & \cdots & 1
\end{array}\right]
$$

Fuente: Adaptado de Toledo et al (2011).

Una vez obtenido las respuestas o decisiones con base en los criterios establecidos se incorporan en la matriz, realizando una suma de manera vertical de los valores numéricos colocados de acuerdo a la escala de comparación (cuadro 1), esta suma permite ponderar cada celda en una matriz adicional, paso seguido se procede a promediar de manera horizontal los valores ponderados, para obtener las porcentajes de evaluación finales como se muestra en el siguiente ejemplo demostrativo:

Cuadro 2. Ejemplo del proceso de ponderación por AHP

\begin{tabular}{|c|c|c|c|c|c|c|c|c|c|}
\hline \multirow[b]{2}{*}{ Criterios } & \multicolumn{4}{|c|}{ Matriz original } & \multicolumn{4}{|c|}{ Matriz ponderada } & \multirow{2}{*}{$\begin{array}{c}\text { Promedio } \\
\text { de los } \\
\text { porcentaje } \\
5 \\
\end{array}$} \\
\hline & $\begin{array}{c}\text { Criterio } \\
1 \\
\end{array}$ & $\begin{array}{c}\text { Criterio } \\
2 \\
\end{array}$ & $\begin{array}{c}\text { Criterio } \\
3 \\
\end{array}$ & $\begin{array}{c}\text { Criterio } \\
4 \\
\end{array}$ & $\begin{array}{c}\text { Criterio } \\
1 \\
\end{array}$ & $\begin{array}{c}\text { Criterio } \\
2 \\
\end{array}$ & $\begin{array}{c}\text { Criterio } \\
3 \\
\end{array}$ & $\begin{array}{c}\text { Criterio } \\
4 \\
\end{array}$ & \\
\hline Criterio 1 & 1 & 5 & 7 & $1 / 3$ & $23.03 \%$ & $44,12 \%$ & $61,76 \%$ & $7.35 \%$ & $34.07 \%$ \\
\hline $\begin{array}{c}\text { Criterio } \\
2\end{array}$ & $1 / 5$ & 1 & 3 & $1 / 5$ & $4,61 \%$ & $8,82 \%$ & $26.47 \%$ & $4,41 \%$ & $11,08 \%$ \\
\hline $\begin{array}{c}\text { Criterio } \\
3\end{array}$ & $1 / 7$ & $1 / 3$ & 1 & 3 & $3,29 \%$ & $2,94 \%$ & $8,82 \%$ & $66,18 \%$ & $20,31 \%$ \\
\hline $\begin{array}{c}\text { Criterio } \\
4 \\
\end{array}$ & 3 & 5 & $1 / 3$ & 1 & $69,08 \%$ & $44,12 \%$ & $2,94 \%$ & $22,06 \%$ & $34.55 \%$ \\
\hline Suma & 4,34 & 11,33 & 11,33 & 4,53 & $100 \%$ & $100 \%$ & $100 \%$ & $100 \%$ & $100 \%$ \\
\hline
\end{tabular}

Como se puede apreciar en el cuadro 2, este método permite llegar a un valor de importancia por criterio de evaluación, utilizado para la toma de decisiones de acuerdo al contexto en que se lleve a cabo la escogencia de criterios, en este caso el criterio 4 es el que tiene mayor ponderación. 
En todo análisis empírico basado diferentes opiniones, pueden existir diferencias o contradicciones en las afirmaciones brindadas, se debe por lo tanto realizar un análisis de inconsistencia, esto con el fin de comprobar que las respuestas son congruentes entre sí, las matrices 2x2 siempre son consistentes, en matrices de $3 \times 3$ es más dificil obtener una consistencia perfecta, debido a la adición de criterios a evaluar, por lo que dicho indicador debe ser de menor o igual a 0,1 para determinar que existe congruencia en las respuestas dadas por el evaluador, los expertos o el grupo de personas entrevistadas, la forma de cálculo es la siguiente:

- Razón de consistencia:

$$
R C=\frac{I C}{I A}
$$

Donde:

IC: Índice de consistencia

IA: Índice aleatorio de consistencia

- Índice de consistencia:

$$
I C=\frac{\lambda \max -n}{n-1}
$$

Donde:

$\lambda$ máx.: Es la sumatoria del resultado de la multiplicación de la matriz original por el vector propio, es decir, el promedio de las ponderaciones obtenidas para cada factor.

n: número de factores de comparación de la matriz

- Índice aleatorio de consistencia:

$$
I A=\frac{1,98 *(n-2)}{n}
$$


Otra forma de calcular este índice es mediante el uso del cuadro 3, que define dicho indicador de acuerdo al número de criterios que se estén analizando y comparando dentro de cada una de las matrices, siendo el utilizado para la presente investigación el procedimiento presentado en la fórmula 4.

Cuadro 3. Índice aleatorio de consistencia de acuerdo al número de atributos a comparar

\begin{tabular}{lllllllllll}
\hline Número de elementos que se comparan & 1 & 2 & 3 & 4 & 5 & 6 & 7 & 8 & 9 & 10 \\
\hline Índice Aleatorio de inconsistencia & 0 & 0 & 0,6 & 0,9 & 1,1 & 1,2 & 1,3 & 1 & 1,5 & 1,5 \\
\hline
\end{tabular}

Fuente: Saaty (2008).

Adicionalmente, es importante destacar que existen diversas aplicaciones para el método AHP como lo son los análisis de sensibilidad (Ishizaka \& Labib, 2011), que permiten reducir el margen de error de las respuestas, al incluir aspectos matemáticos para mejorar las ponderaciones, una de estas formas es multiplicar la matriz original "n" cantidad de veces por ella misma, hasta que el ponderador final deje sufrir cambios, utilizado para el presente estudio para disminuir la sensibilidad en las ponderaciones finales.

\section{Resultados}

\section{Elección de criterios y subcriterios de riesgo operativo en agricultura}

Para la selección de criterios y subcriterios se utilizó el concepto de riesgo operativo definido por Quirós (2018) donde se especifica que las fuentes (criterios) de riesgo operativo son los procesos productivos, la gestión del recurso humano, la gestión de las tecnologías productivas, y los factores climáticos y ambientales, mientras que se seleccionaron dentro de cada fuente de riesgo una serie de factores (subcriterios) detalladas en la figura 1, donde, de acuerdo al criterio de experto se pueden presentar eventos que generen riesgo en los sistemas agrícolas. La caracterización de cada fuente y factor de riesgo se puede consultar de acuerdo a la propuesta definida por Quirós-Badilla (2019). 


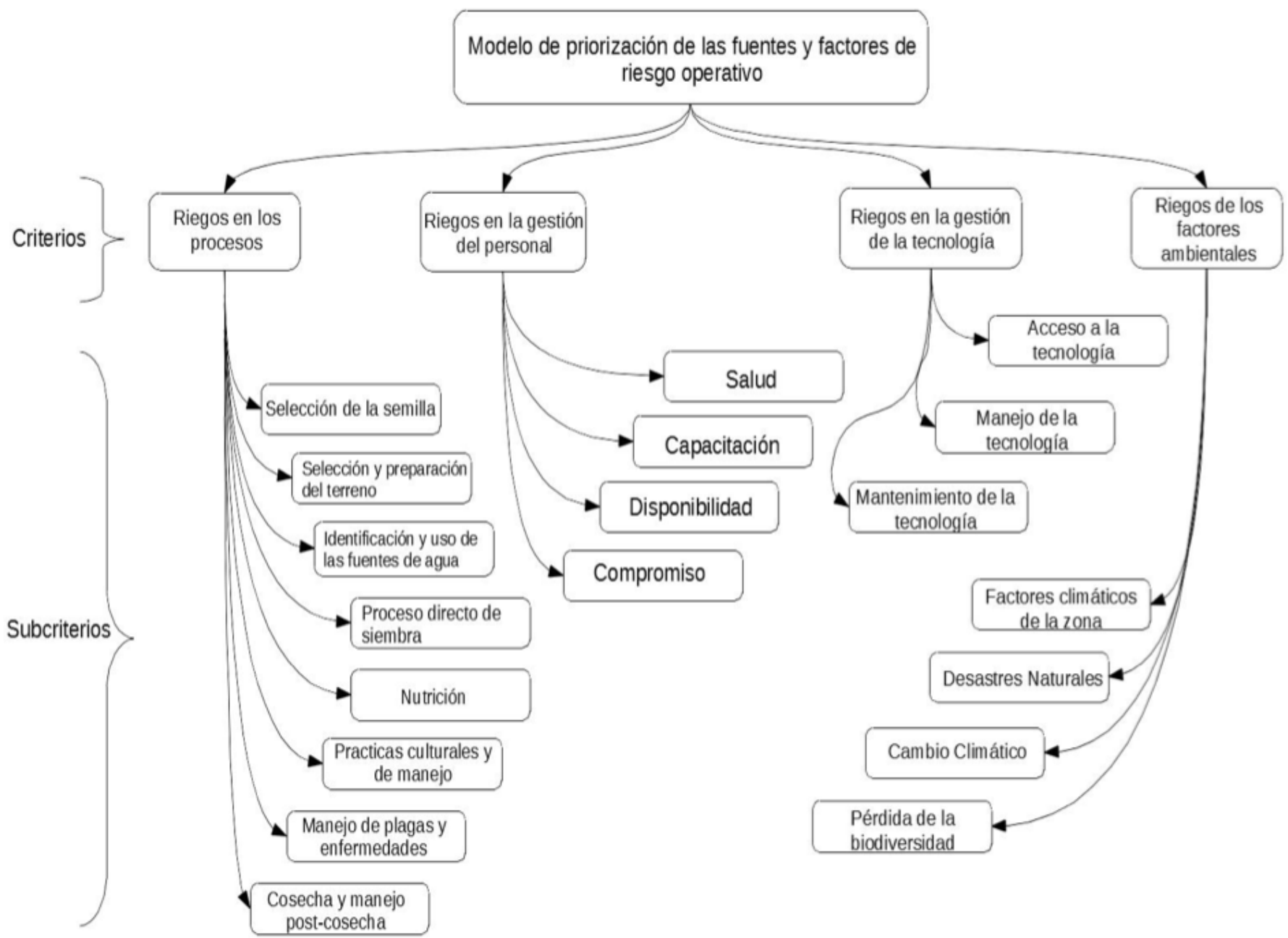

Figura 1. Estructura del modelo de decisión por el método de análisis jerárquico analítico

Fuente: Adaptado de Quirós (2018)

\section{Jerarquización de las fuentes y factores de riesgo operativo en agricultura}

Por medio de esta estructura de criterios y subcriterios para cada fuente de riesgo operativo aplicando el método AHP, se logró obtener una ponderación de importancia con respecto al riesgo de cada fuente de riesgo operativo y de cada factor dentro de cada una de ellas, en una base de $100 \%$, como se muestra en el cuadro 4. Así mismo, estos factores permiten obtener información sobre aspectos, procesos o situaciones que son críticas en la agricultura de manera puntual y documentada. 
El proceso de recolección de información con expertos permitió comparar cada fuente de riesgo de manera pareada por medio de una matriz 4X4, los factores en los procesos productivos por medio de una matriz 8x8, una matriz 4X4 para los factores en la gestión del recurso humano, una matriz 3×3 para los factores en la gestión de las tecnologías de producción y una matriz 4X4 para los factores climáticos y ambientales, analizando en cada una de las matrices su indicador de consistencia, para determinar congruencia en las respuestas brindadas por los expertos.

Cuadro 4. Ponderación de las fuentes y factores de riesgo operativo en agricultura

\begin{tabular}{|c|c|c|c|}
\hline $\begin{array}{c}\text { Fuentes de Rlesgo } \\
\text { Operativo }\end{array}$ & Ponderación & Factores de riesgo & Ponderación \\
\hline \multirow{8}{*}{ Procesos } & \multirow{8}{*}{$2495 \%$} & Selección del material de siembra & $1352 \%$ \\
\hline & & Selección y preparación del terreno & $3.81 \%$ \\
\hline & & $\begin{array}{l}\text { Identificación y uso de las fuentes de } \\
\text { agua }\end{array}$ & $24.54 \%$ \\
\hline & & Siembra & $5.62 \%$ \\
\hline & & Nutrición & $14.40 \%$ \\
\hline & & Prácticas culturales o de manejo & $5.62 \%$ \\
\hline & & Manejo de plagas y enfermedades & $27.99 \%$ \\
\hline & & Cosecha y manejo post cosecha & $4,49 \%$ \\
\hline \multirow{4}{*}{$\begin{array}{l}\text { Gestión y manejo del } \\
\text { personal }\end{array}$} & \multirow{4}{*}{$9.55 \%$} & Salud & $7.52 \%$ \\
\hline & & Capacitación & $25.53 \%$ \\
\hline & & Disponibilidad & $50,83 \%$ \\
\hline & & Compromiso & $4.49 \%$ \\
\hline \multirow{3}{*}{$\begin{array}{l}\text { Gestión y manejo de la } \\
\text { tecnologia }\end{array}$} & \multirow{3}{*}{$9.55 \%$} & Acceso a la Tecnologia & $42,86 \%$ \\
\hline & & Manejo de la tecnologia & $14,29 \%$ \\
\hline & & Mantenimiento & $42,86 \%$ \\
\hline \multirow{4}{*}{$\begin{array}{l}\text { Factores climáticos y } \\
\text { ambientales }\end{array}$} & \multirow{4}{*}{$55.96 \%$} & Factores climáticos de la zona & $53.83 \%$ \\
\hline & & Desastres naturales y catástrofes & $30,51 \%$ \\
\hline & & Cambio Climático & $7,83 \%$ \\
\hline & & Pérdida de la Biodiversidad & $7,83 \%$ \\
\hline \multicolumn{3}{|c|}{ Razón de consistencia matriz $4 \times 4$ fuentes de riesgo } & $0,0163<0,1$ \\
\hline \multicolumn{3}{|c|}{ Razón de consistencia matriz $8 \times 8$ factores de riesgo en los procesos } & $0,0340<0,1$ \\
\hline \multicolumn{3}{|c|}{ Razón de consistencia matriz $4 \times 4$ factores de riesgo en la gestión del personal } & $0,0754<0,1$ \\
\hline \multicolumn{3}{|c|}{ Razón de consistencia matriz $3 \times 3$ factores de riesgo en la gestión de tecnologías } & $0,0000<0,1$ \\
\hline \multicolumn{3}{|c|}{ Razón de consistencia matriz $4 \times 4$ factores de riesgo climáticos y ambientales } & $0,0579<0,1$ \\
\hline
\end{tabular}


El resultado de la aplicación del método AHP muestra consistencia en las respuestas de los expertos en la jerarquización de la importancia con respecto al riesgo de las fuentes y factores de riesgo operativo en sistemas de producción agrícola con cada una de las matrices por debajo del indicador de 0,1; siendo los factores climáticos y ambientales como los más riesgosos con una ponderación del 55,96\% del total, seguido de los procesos productivos con un 24,95\% del total para las fuentes de riesgos, mientras que la gestión del recurso humano y de las tecnologías de producción representa el mismo peso en relación al riesgo con un 9.55\%.

En relación a los factores de riesgo dentro de cada fuente, el manejo de plagas y enfermedades (27,99\%) y la identificación y uso de las fuentes de agua (24,54\%) resultan ser las 2 etapas del proceso productivo que generan mayores riesgos en la producción agrícola. Por su parte, dentro de la gestión del recurso humano la disponibilidad de mano de obra es el factor más riesgoso con un 50,83\%, el acceso a tecnología y mantenimiento de la tecnología cada una con 42,86\% con respecto al riesgo son los factores más influyentes en la gestión de las tecnologías de producción. Para la última fuente, los factores climáticos de la zona se caracterizan como los más riesgosos, seguido de los desastres naturales y catástrofes con un 30,51\% del total de esta fuente de riesgo, la pérdida de la biodiversidad y cambio climático tienen la misma ponderación de un 7,83\%.

\section{Identificación de eventos riesgosos. Caso de estudio en el cultivo de café (coffea arabica)}

Con la ejecución del segundo panel de expertos en el cultivo de café, se utilizaron estás mismas fuentes y factores de riesgo operativo en agricultura para identificar eventos especíicos en el cultivo de café por medio del diagrama de criterios y sub-criterios definido por el método AHP, categorizándolas de manera puntual dentro de cada factor de riesgo en el proceso productivo, gestión del recurso humano, gestión de tecnologías productivas y factores climáticos y ambientales.

El cuadro de identificación de eventos de riesgos se representa en el anexo 1 de manera puntual. Para este proceso se decidió establecer acontecimientos de manera generalizada dentro del contexto nacional del cultivo de café, donde se logró identificar 79 eventos de riesgo representado en el cuadro 5. 


\begin{tabular}{|c|c|c|}
\hline Fuentes de riesgo & Factores de riesgo & $\begin{array}{l}\text { Cantidad de eventos } \\
\text { de riesgo identificados }\end{array}$ \\
\hline \multirow{8}{*}{$\begin{array}{l}\text { Eventos en los } \\
\text { orocesos } \\
\text { productivos (33) }\end{array}$} & Selección del material de siembra & 3 \\
\hline & Selección y presaración del terreno & 4 \\
\hline & Identificoc ón y uso de wa fuentes de ague & 4 \\
\hline & Proresos dires:lo de sientura & 5 \\
\hline & Nutricón & 5 \\
\hline & Präcticas culturales y de manejo & 6 \\
\hline & Manojo de plagas y enfermedades & 3 \\
\hline & Cosecho y manejo postcosecha & 3 \\
\hline \multirow{4}{*}{$\begin{array}{l}\text { Fyeriless britid } \\
\text { gestion dal } \\
\text { recurso humbermo } \\
\text { (16) }\end{array}$} & 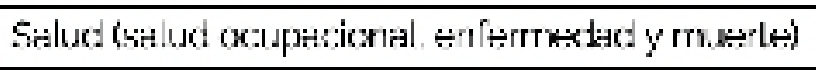 & 4 \\
\hline & Canacitacjón & 4 \\
\hline & Disporibilidad & 2 \\
\hline & Comproniso & 6 \\
\hline \multirow{3}{*}{$\begin{array}{l}\text { Eventos en la } \\
\text { gestión de bis } \\
\text { texndogias de } \\
\text { producc on (15) }\end{array}$} & Acceso a lecrodog as de orccluccićn & 5 \\
\hline & Manejo de la Lecrnolocjie & 5 \\
\hline & Manterimiento & 5 \\
\hline \multirow{4}{*}{$\begin{array}{c}\text { Eventos en los } \\
\text { fackores } \\
\text { climàticosy } \\
\text { ambiontales (15) }\end{array}$} & Factcres climàticos comunes en la regicn & 3 \\
\hline & Dosicstres noturales o cathistrofos & 5 \\
\hline & Carnbic clirrúlico & 3 \\
\hline & Pérdida de la biodiversidad & 4 \\
\hline
\end{tabular}

Se hace énfasis que dentro de cada factor de riesgo, pueden existir más o menos sucesos de los señalados, dependiendo del contexto individualizado de cada caso en particular, esta identificación representa ejemplos recurrentes de eventos con carácter riesgoso en el cultivo de café para propósito de la aplicación de una gestión administrativa de riesgos y que sirven en una primera etapa de evaluación de riesgos, que de acuerdo al contexto del sistema cafetalero pueden ampliarse o disminuirse.

\section{Discusión}

Así como lo comprueban Toledo et al (2011), el uso del método AHP para jerarquizar factores de riesgo en agricultura resulta útil para determinar estrategias de producción, comercialización o gestión administrativa del sistema productivo, por este motivo resulta importante ponderar variables de riesgo sobre las cuales el agricultor posee cierto nivel de control o sobre las cuales puede tomar medidas de reducción de riesgo, como lo es caso del riesgo operativo. 
Este análisis permitió priorizar áreas donde se deben de jerarquizar las decisiones para evitar pérdidas significativas en la producción. A su vez, es la base para la generación de sistemas administrativos de los riesgos en la agricultura, ya que permite la creación de índices o indicadores de gestión. Este método es además ampliamente utilizado en otros campos (ingeniería, medicina, física, psicología y otros) donde acuerdo con Zyoud y Fuchs-Hanusch (2017) en el área de las ciencias agrícolas y biológicas se han publicado más de 900 documentos con su aplicación.

La utilización de paneles de expertos especializados en la gestión de riesgos en agricultura permite reducir la inconsistencia en las respuestas una vez aplicado el método AHP, ya que esto se presenta como una de las limitantes del modelo dada la independencia de respuestas, es decir, debe de existir consenso en las contestaciones; no obstante de acuerdo con otras investigaciones (Ishizaka \& Labib, 2011; Zyoud \& Fuchs-Hanusch, 2017) se evidencia como un modelo matemático útil para la toma de decisiones al tener aplicaciones para reducir la sensibilidad una vez obtenidas las ponderaciones.

Los resultados de la presente investigación señalan a los factores ambientales y climáticos como los más riesgosos dentro de un sistema agrícola en concordancia con las afirmaciones de otras investigaciones (Elias et al., 2019; Pelka et al., 2015), principalmente porque algunos eventos específicos de este tipo son impredecibles y el tiempo de reacción es reducido, siendo el factor donde más acciones y planeación deben tomar los productores, asi como la cantidad de recursos financieros que deben destinar para enfrentar estos riesgos.

Estos resultados se pueden comparar con los obtenidos por Toledo et al (2011), donde se obtuvo que el factor con mayor peso (por medio del método AHP) fue la variación en los precios y costos directos de los productos, el clima en este estudio fue catalogado como el factor menos riesgoso de los cuatro analizados, principalmente porque se limitó a las variables de sequia y viabilidad de irrigación artificial, y no tomó en cuenta aspectos como desastres naturales, cambio climático o pérdida de la biodiversidad, sin embargo, lo clasifican como el más riesgoso en el ámbito de la percepción del productor.

Cabe destacar el aspecto ambiental como uno de los más importantes en agricultura, en la línea de los resultados de este estudio y en la generación de resiliencia antes diversos eventos de riesgo en el sector agrícola, se puede mencionar lo manifestado por Srinivasa Rao et al (2019) en el análisis de las dimensiones ambiental, económica, social e institucional por medio del método AHP, siendo la dimensión ambiental la más importante para gestionar acciones de mitigación y resiliencia en agro ecosistemas frente al cambio climático.

Por otra parte, el proceso productivo es de principal cuidado ya que algunas etapas son críticas en cuidados y manejos al presentarse factores o eventos exógenos que afectan los cultivos como las plagas y las enfermedades y donde además se deben administrar recursos para garantizar la disponibilidad de semilla e insumos productivos (Fahad et al., 2018). Las gestión del recurso humano y de las tecnologías productivas poseen el menor peso de riesgo, ya que 
se caracterizan por tener un control administrativo casi total y donde las decisiones de manejo son mayormente controladas, a pesar de que el recurso monetario es importante en su gestión, Toledo et al (2011) destaca el riesgo humano segundo lugar dentro de la ponderación realizada, principalmente por el costo y el nivel de conocimiento necesario de las labores productivas por parte de los trabajadores.

Un segundo panel de experto de un cultivo en particular, en este caso el café (coffea arabica), resultó útil para identificar eventos de riesgo específicos en cada uno de los factores de riesgos propuestos, analizados desde el enfoque operativo de la producción, bajo la estructura determinada por el método AHP, los cuales pueden ser evaluados de manera individualizada por medio de matrices de calor, analizando la frecuencia en que se presentan, el impacto posible que pueden ocasionar y la gestión para la mitigación o reducción de los posibles efectos, explicando de manera más amplia el efecto en cada sistema cafetalero en particular, como también lo destacan Palma Rodríguez (2011) en sector bancario y Quirós-Badilla (2019) en el área agrícola. Este modelo de identificación puede ser utilizado en la evaluación de riesgos específicos en otros cultivos.

\section{Conclusiones y recomendaciones}

De acuerdo con el análisis teórico, el riesgo operativo como tal no se ha analizado de manera profunda en el sector agropecuario, la presente investigación ofrece una ponderación generalizada para el riesgo operativo en sistemas de producción agrícola, que resulta útil para desarrollar una base en la gestión administrativa de riesgos dentro de un sistema agrario, al abarcar los procesos productivos, la gestión del recurso humano, la gestión de las tecnologías de producción y factores climáticos y ambientes, siendo un concepto más integral y utilizado ampliamente en los campos financieros y empresariales.

La identificación de eventos específicos de riesgos permitió generar una base para la evaluación de riesgos dentro del concepto del riesgo operativo en la agricultura, es este caso en el cultivo de café (pero replicable en otros cultivos), como se mencionó pueden existir más o menos variables de las señaladas, que dependen de las situaciones específicas del sistema agrícola evaluado, pero que permiten una evaluación integral con la adición de matrices de calor analizando la frecuencia, el impacto y la gestión de riesgos presente.

Este ejercicio por medio de fuentes y factores de riesgo operativo ponderadas y eventos evaluados por medio de matrices de calor pueden permitir la creación de un sistema integral de evaluación de riesgos en próximas investigaciones, donde se presenten resultados comparables por medio de índices e indicadores, además de ampliar una rama de investigación importante en sector agropecuario en la generación de nuevo conocimiento y metodologías para la gestión del riesgo.

La línea de investigación del riesgo en el sector agricola es amplia de manera teórica, sin embargo, de manera práctica las aplicaciones o herramientas a utilizar no están muy bien defini- 
das y no siempre son aplicables a la amplia diversidad de sistemas productivos, aumentar la investigación en este campo es importante, siendo una necesidad y oportunidad para futuros estudios, así como la aplicación de evaluaciones en el cultivo de café en un primer ámbito, y ampliarse con otros campos como el informático, para crear herramientas al alcance de los productores, y obtener datos para mejorar las políticas públicas en materia de riesgo agrícola.

Los resultados mostraron la identificación de una gran cantidad de eventos que generan riesgo en el cultivo de café, algunos podrian ser más dañinos que otros, para lo cual se requiere ampliar a métodos de evaluación en este aspecto en el área de la investigación de cultivo, sin embargo, es importante transmitirlos a los productores para que gestionen aquellos eventos presentes en sus sistemas productivos. Además, las instituciones involucradas en el cultivo de café deben fortalecer los proyectos dirigidos a la gestión de riesgos, no solo para evitarlos, si no para desarrollar mecanismos de acción en caso de que presenten situaciones en diferentes escalas.

\section{Literatura citada}

Arbeláez, J. C., Franco, L. C., Betancur, C., Murillo, J. G., Andrea, P., Henao, V. M., Londoño, J. A., Mejía, C. M., Marcela, D., Salazar, E., Salazar, L. F., Valderrama, N., \& Varela, D. C. (2006). Riesgo operacional: Reto actual de las entidades financieras. Revista Ingenierias Universidad de Medellín, 5, 14.

Badri, A., Nadeau, S., \& Gbodossou, A. (2013). A new practical approach to risk management for underground mining project in Quebec. Journal of Loss Prevention in the Process Industries, 26(6), 1145-1158. https://doi.org/10.1016/j.jlp.2013.04.014

Baquet, A. E., Hambleton, R., \& Jose, D. (1997). Introduction to risk management. http://agris. fao.org/agris-search/search.do?recordID=US201300017609

Barnett, B. J., \& Coble, K. H. (2008). Are Our Agricultural Risk Management Tools Adequate for a New Era? Choices, 1, 36-39. https://doi.org/10.22004/ag.econ.94700

Bergfjord, O. J. (2009). Risk perception and risk management in Norwegian aquaculture. Journal of Risk Research, 12(1), 91-104. https://doi.org/10.1080/13669870802488941

Delfiner, M., \& Pailhé, C. (2009, enero 5). Técnicas cualitativas para la gestión del Riesgo Operacional [MPRA Paper]. https://mpra.ub.uni-muenchen.de/15809/

Dos Santos, P. H., Neves, S. M., Sant'Anna, D. O., Oliveira, C. H. de, \& Carvalho, H. D. (2019). The analytic hierarchy process supporting decision making for sustainable development: An overview of applications. Journal of Cleaner Production, 212, 119-138. https://doi.org/10.1016/j. jclepro.2018.11.270 
Elias, E. H., Flynn, R., Idowu, O. J., Reyes, J., Sanogo, S., Schutte, B. J., Smith, R., Steele, C., \& Sutherland, C. (2019). Crop Vulnerability to Weather and Climate Risk: Analysis of Interacting Systems and Adaptation Efficacy for Sustainable Crop Production. Sustainability, 11(23), 6619. https://doi.org/10.3390/su11236619

Fahad, S., Wang, J., Khan, A. A., ullah, A., Ali, U., Hossain, M. S., Khan, S. U., Huong, N. T. L., Yang, X., Hu, G., \& Bilal, A. (2018). Evaluation of farmers' attitude and perception toward production risk: Lessons from Khyber Pakhtunkhwa Province, Pakistan. Human and Ecological Risk Assessment: An International Journal, 24(6), 1710-1722. https://doi.org/10.1080/10807039.201 8.1460799

Flaten, O., Lien, G., Koesling, M., Valle, P. S., \& Ebbesvik, M. (2005). Comparing risk perceptions and risk management in organic and conventional dairy farming: Empirical results from Norway. Livestock Production Science, 95(1-2), 11-25. https://doi.org/10.1016/j.livprodsci.2004.10.014

Girdžiūtè, L. (2012). Risks in Agriculture and Opportunities of their Integrated Evaluation. Procedia - Social and Behavioral Sciences, 62, 783-790. https://doi.org/10.1016/j.sbspro.2012.09.132

Guan, Z., \& Wu, F. (2017). Modeling heterogeneous risk preferences. Agricultural Finance Review, 77(2), 324-336. https://doi.org/10.1108/AFR-03-2015-0016

Hansson, H., \& Lagerkvist, C. J. (2012). Measuring farmers' preferences for risk: A domain-specific risk preference scale. Journal of Risk Research, 15(7), 737-753. https://doi.org/10.1080/13 669877.2012 .657217

Harwood, J., Heifner, R., Coble, K., Perry, J., \& Somwaru, A. (1999). Managing Risk in Farming: Concepts, Research, and Analysis. U.S. Department of Agriculture, ERS.

He, B., Wang, Q.-J., Wu, D., \& Zhou, B.-B. (2016). Spatio-temporal characteristics of agricultural drought in Shaanxi Province, China based on integrated disaster risk index. Chinese Journal of Applied Ecology, 27(10), 3299-3306. Scopus. https://doi.org/10.13287/j.1001-9332.201610.040

Ishizaka, A., \& Labib, A. (2011). Review of the main developments in the analytic hierarchy process. Expert Systems with Applications. https://doi.org/10.1016/j.eswa.2011.04.143

Kahan, D. (2013). Managing risk in farming (Reprint). FAO.

Komarek, A. M., De Pinto, A., \& Smith, V. H. (2020). A review of types of risks in agriculture: What we know and what we need to know. Agricultural Systems, 178, 102738. https://doi.org/10.1016/j.agsy.2019.102738 
Lagerkvist, C. J. (2005). Assessing farmers' risk attitudes based on economic, social, personal, and environmental sources of risk: Evidence from Sweden. Selected Paper 136315. https:// doi.org/10.22004/ag.econ.19361

Li, L., Kuang, Z., Mo, J., \& Meng, C. (2013). Assessment of risk ranking for autumn drought in Guangxi province based on AHP and GIS. Nongye Gongcheng Xuebao/Transactions of the Chinese Society of Agricultural Engineering, 29(19), 193-201. Scopus. https://doi.org/10.3969/j. issn.1002-6819.2013.19.024

Lu, T., Guo, J., Cheng, M., Li, H., Aimaiti, Y., \& Liu, Y. (2016). Model establishment and zoning of wind-dust risk assessment to featured forestry and fruit industry in Xinjiang. Nongye Gongcheng Xuebao/Transactions of the Chinese Society of Agricultural Engineering, 32, 169-176. Scopus. https://doi.org/10.11975/j.issn.1002-6819.2016.z2.023

Miller, A., Dobbins, C., Pritchett, J., Boehlje, M., \& Ehmke, C. (2004). Risk management for farmers (p. 31). Departamento de Economía Agrícola. Universidad de Purdue. https://www. researchgate.net/publication/23514946_RISK_MANAGEMENT_FOR_FARMERS

Palma Rodríguez, C. (2011). ¿Cómo construir una matriz de riesgo operativo? Ciencias Económicas, 29(1), 630-635.

Pelka, N., Musshoff, O., \& Weber, R. (2015). Does weather matter? How rainfall affects credit risk in agricultural microfinance. Agricultural Finance Review, 75(2), 194-212. https://doi. org/10.1108/AFR-10-2014-0030

Quirós Badilla, D. F. (2018). Propuesta de una metodología para la evaluación de riesgo operativo en sistemas de producción agrícola en Costa Rica aplicado a un estudio de caso en el cultivo de café (Coffea arabica) [Tesis de Licenciatura]. Universidad de Costa Rica.

Quirós-Badilla, D. F. (2019). Riesgo operativo en sistemas de producción agrícola. Propuesta base para su evaluación. Revista e-Agronegocios, 5(2). https://doi.org/10.18845/rea.v5i2.4455

Rossinskaya, M. V., Saveleva, N. A., \& Rokotyanskaya, V. V. (2019). Tools for improving operational risk management in agriculture. Iraqi Journal of Agricultural Sciences, 50(Special Issue), 134-142. Scopus.

Saaty, T. L. (2008). Decision making with the analytic hierarchy process. International Journal of Services Sciences, 1(1), 83. https://doi.org/10.1504/IJSSCI.2008.017590

Srinivasa Rao, C., Kareemulla, K., Krishnan, P., Murthy, G. R. K., Ramesh, P., Ananthan, P. S., \& Joshi, P. K. (2019). Agro-ecosystem based sustainability indicators for climate resilient agriculture in India: A conceptual framework. Ecological Indicators, 105, 621-633. Scopus. https://doi. org/10.1016/j.ecolind.2018.06.038 
Sulewski, P., \& Kłoczko-Gajewska, A. (2014). Farmers' risk perception, risk aversion and strategies to cope with production risk: An empirical study from Poland. Studies in Agricultural Economics, 116(3), 140-147. https://doi.org/10.7896/j.1414

Toledo, R., Engler, A., \& Ahumada, V. (2011). Evaluation of Risk Factors in Agriculture: An Application of the Analytical Hierarchical Process (AHP) Methodology. Chilean Journal of Agricultural Research, 71(1), 114-121. https://doi.org/10.4067/S0718-58392011000100014

Wilson, P. N., Luginsland, T. R., \& Armstrong, D. V. (1988). Risk Perceptions and Management Responses of Arizona Dairy Producers. Journal of Dairy Science, 71(2), 545-551. https://doi. org/10.3168/jds.S0022-0302(88)79588-0

Yalçinkaya, S. (2014). Agricultural Risk Management: Optimization Model Proposal. En Proceedings of MAC-EMMT 2014: The 3rd Multidisciplinary Academic Conference in Prague Economics, Management, Marketing and Tourism MAC-EMMT 2014. MAC Prague consulting.

Young, D. L. (1979). Risk Preferences of Agricultural Producers: Their Use in Extension and Research. American Journal of Agricultural Economics, 61(5), 1063. https://doi. org/10.2307/3180376

Zyoud, S. H., \& Fuchs-Hanusch, D. (2017). A bibliometric-based survey on AHP and TOPSIS techniques. Expert Systems with Applications, 78, 158-181. https://doi.org/10.1016/j. eswa.2017.02.016 


\section{Anexos}

\section{Anexo 1. Identificación de eventos de riesgo operativo en el cultivo de café en Costa Rica}

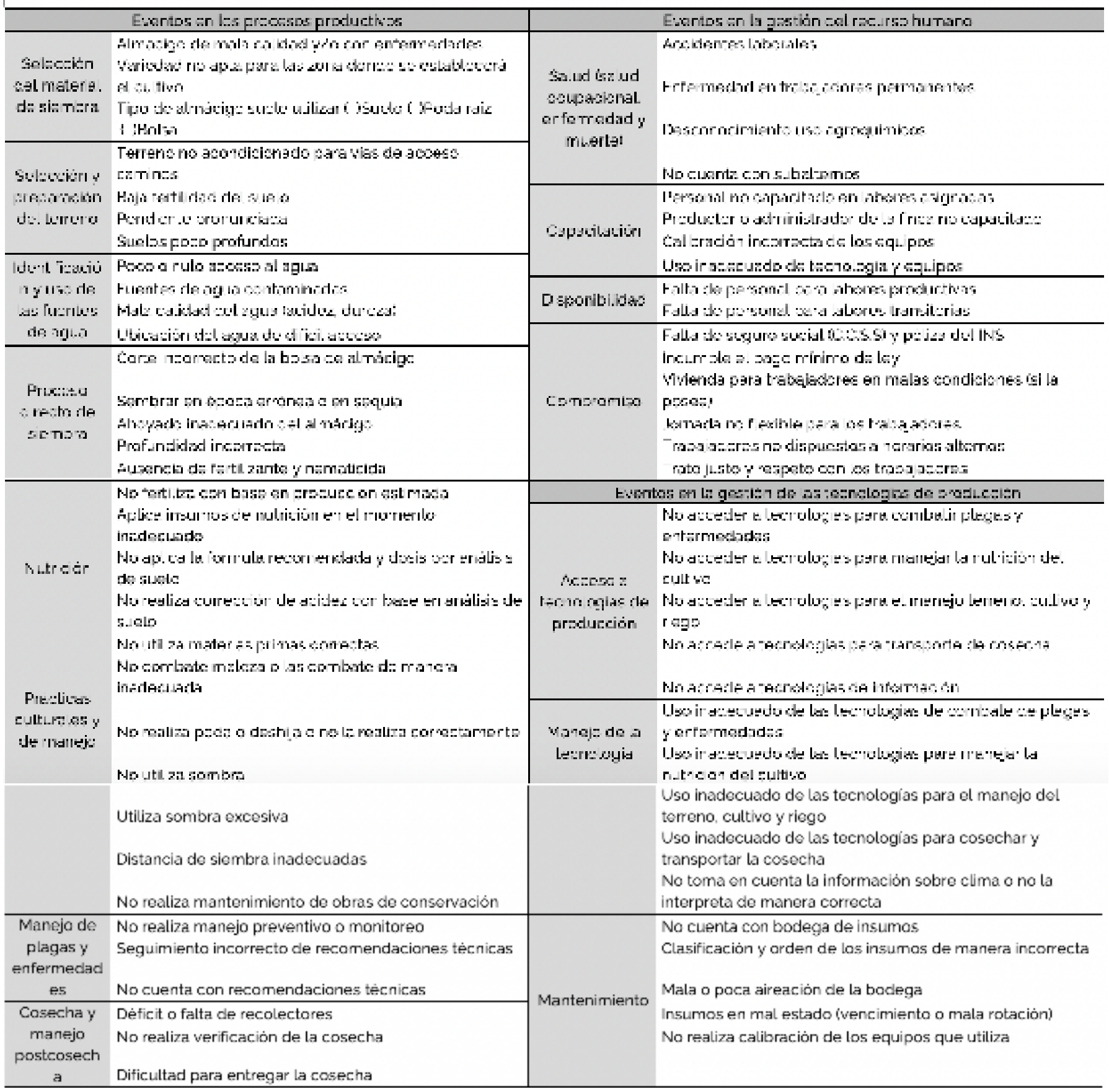




\begin{tabular}{|c|c|}
\hline \multicolumn{2}{|c|}{ Eventos en los factores climaticos y ambientales } \\
\hline Factores climàticos comunes en la región & $\begin{array}{l}\text { Afectación en el cultivo por altas o bajas temperaturas } \\
\text { Afectación en el cultivo por déficit o exceso de luvias } \\
\text { Afectación del cultivo por exceso de viento }\end{array}$ \\
\hline Desastres naturales o catàstrofes & $\begin{array}{l}\text { Afectación sobre el cultivo por terremotos } \\
\text { Afectación sobre el cultivo por deslizamientos } \\
\text { Afectación en el cultivo por huracanes } \\
\text { Afectación en el cultivo por cierre de caminos debido a fenómenos } \\
\text { naturales } \\
\text { Afoctación sobre el cultivo por la caída de granizo }\end{array}$ \\
\hline Cambio climático & $\begin{array}{l}\text { Afoctación del cultivo por sequia } \\
\text { Afoctación el cultivo por saturación de agua en los sue los } \\
\text { Desplazamiento de la producción a zonas más altas }\end{array}$ \\
\hline Pórdida de la biodiversidad & $\begin{array}{l}\text { Afoctación en el cultivo por pérdida de polinizadores } \\
\text { Pérdida de la biodiversidad on el suelo } \\
\text { Pérdida de la cobertura boscosa on la finca } \\
\text { Pérdida de controladores bio lógicos de plagas }\end{array}$ \\
\hline
\end{tabular}


